Research Article

\title{
Low Frequency of MKRN3 and DLK1 Variants in Chinese Children with Central Precocious Puberty
}

\author{
Ting Chen $\mathbb{D}^{1},{ }^{1}$ Linqi Chen $\mathbb{D}^{1},{ }^{1}$ Haiying Wu $\mathbb{D}^{1},{ }^{1}$ Rongrong Xie $\mathbb{D}^{1},{ }^{1}$ Fengyun Wang $\left(\mathbb{D},{ }^{1}\right.$ \\ Xiuli Chen $(\mathbb{D}){ }^{1}$ Hui Sun $\left(\mathbb{D},{ }^{1}\right.$ and Fei Xiao $\mathbb{D}^{2}$ \\ ${ }^{1}$ Department of Endocrinology, Genetics and Metabolism, Children's Hospital of Soochow University, Suzhou, Jiangsu, China \\ ${ }^{2}$ School of Basic Medicine \& Biological Sciences, Medical College of Soochow University, Soochow University, Suzhou, \\ Jiangsu, China
}

Correspondence should be addressed to Ting Chen; ct1596@126.com and Fei Xiao; xiaofei@suda.edu.cn

Received 16 January 2019; Revised 20 July 2019; Accepted 18 September 2019; Published 3 October 2019

Academic Editor: Alberto Ferlin

Copyright (c) 2019 Ting Chen et al. This is an open access article distributed under the Creative Commons Attribution License, which permits unrestricted use, distribution, and reproduction in any medium, provided the original work is properly cited.

Background. Central precocious puberty (CPP) is defined by gonadotropin-dependent development of secondary sexual characteristics before the age of 8 years in girls and 9 years in boys. MKRN3 and DLK1 are two genes, disease-causing variants of which have recently been discovered to cause idiopathic CPP. Methods. We screened 173 Chinese patients ( 9 males and 164 females; 9 familial and 164 sporadic) with ICPP and 43 patients ( 9 males and 34 females; 3 familial and 40 sporadic) with early puberty for variants in MKRN3. We also screened 19 patients with ICPP and early puberty for variants of DLK1 (17 males and 2 females; 5 familial and 14 sporadic). Results. We identified four novel missense variants of MKRN3, c.1138G > A (p.Glu380Lys), c.1420T > A (p.Leu474Met), c.673C > G (p.Leu225Val), and c.1071C > G (p.Ile357Met) in two sporadic cases and three familial cases. According to ACMG standards, two MKRN3 variant (p.Glu380Lys and p.Ile357Met) are likely pathogenic, and two others are of uncertain significance. We also performed bioinformatic analysis to evaluate the impact of variants on MKRN3 protein structures, which showed that Ile357Met locates at the zinc-binding region (C3HC4 RING finger motif), while Glu380Lys is spatially extremely close to the C3HC4 RING finger, MKRN-specific Cys-His domain, and the third C3H1 zinc-finger motif region. Per Glu380Lys, Glu with negative charges has been changed into Lys with positive charges, which may affect the hydrogen bond formation between amino acids and the stability of the local structure, thus affecting the binding of zinc iron to MKRN3 protein. Besides, we did not identify any variants of DLK1 gene in our patients. Conclusions. In this study, we report four novel $M K R N 3$ variants in patients with ICPP. Moreover, we did not find any variants of $D L K 1$ gene. Variants of MKRN3 are relatively uncommon in Chinese ICPP patients.

\section{Introduction}

Central precocious puberty (CPP) is defined pathophysiologically by the premature activation of the hypothalamicpituitary-gonadal axis and clinically by the development of secondary sexual characteristics before the age of 8 years in girls and 9 years in boys [1]. CPP can be either idiopathic (ICPP) or secondary to CNS lesions. ICPP is much more common compared to secondary CPP, and one-third of ICPP was reported to be familial [2]. Early puberty is usually defined by the age of pubertal onset between 8 and 9 years old in girls and between 9 and 10 years old in boys [3].
To date, only deleterious variants in four genes have been reported as causes of CPP [4-7], including KISS1, KISS1R, $M K R N 3$, and DLK1. KISS1 and KISS1R gene defects caused CPP is extremely rare. Besides, the polymorphisms of KISS1 gene have also been associated with CPP $[8,9]$. In 2013, through a series of familial aggregation studies, Abreu et al. discovered that pathogenic variants of the gene encoding makorin RING finger protein 3 (MKRN3) would cause CPP [6]. Since then, about 33 pathogenic variants in MKRN3 gene have been discovered in CPP patients (Figure 1) $[10,11]$. Accumulating evidences showed that pathogenic variants in MKRN3 are the most frequent genetic cause of 


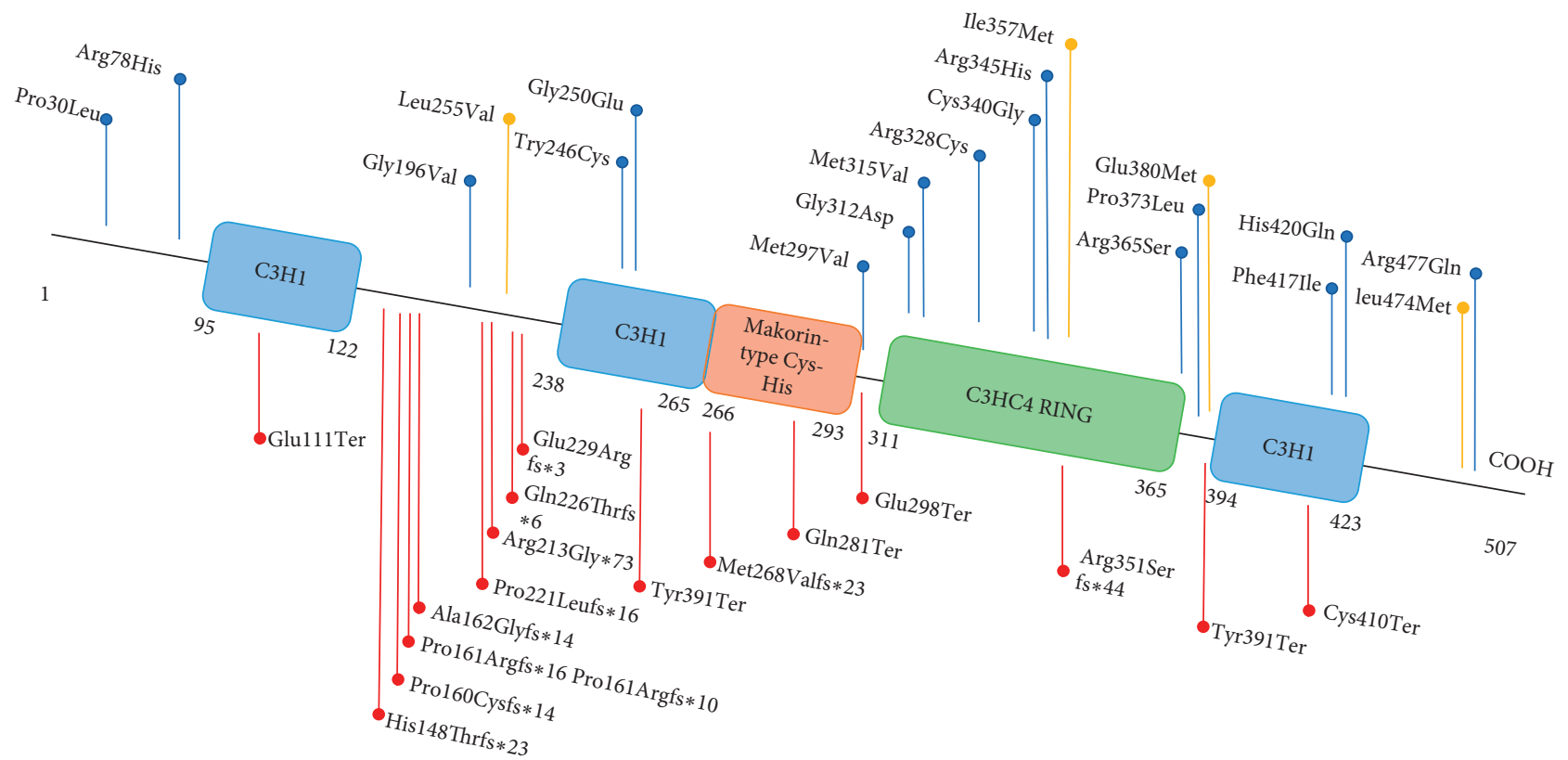

FIGURE 1: Location of MKRN3 variants identified in patients with central precocious puberty. Blue boxes, zinc-finger domains; red box, makorin-type Cys-His motif; green box, C3HC4 RING finger motif. Red arrows indicate the locations of all reported variants which will cause nonsense or frameshift variants. Blue arrows indicate the locations of all reported missense variants, and yellow arrows indicate missense variants we found in the present study.

familial ICPP [10]. In 2017, Dauber et al. identified a genetic defect in DLK1 gene, which encodes delta-like 1 homolog, associating with familial ICPP [7]. MKRN3 and DLK1 both locate within imprinted loci which were previously revealed to be associated with human puberty initiation through large genome-wide studies [12].

In this study, we screened $M K R N 3$ and $D L K 1$ variation in a group of Chinese patients with either ICPP or early puberty, in order to explore the frequency of variants of both genes in Chinese patients.

\section{Material and Methods}

2.1. Subjects. The patients reported in this study attended the Endocrinology, Genetics, and Metabolism Department of Children's Hospital of Soochow University (Suzhou, China) between September 1, 2015, and May 31, 2018. A total of 173 patients (9 males and 164 females; 9 familial and 164 sporadic) with ICPP and 43 patients ( 9 males and 34 females; 3 familial and 40 sporadic) with early puberty were enrolled in this study. All the patients involved in this study are Chinese. Patients with CPP secondary to central nervous system pathology (i.e., tumors or nonspecific cerebral anomalies associated with CPP) were excluded from the study.

Informed consent was obtained from the patients and family members included in this study, following procedures specified by the Ethics Committee of Children's Hospital of Soochow University.

2.2. Study Design. All the participants underwent clinical examination, blood sampling, bone age, and brain MRI. The patients had been diagnosed with ICPP (pubertal onset before age 8 in girls and before age 9 in boys) or early puberty (pubertal onset in girls $\geq 8$ years but $<9$ years old or pubertal onset in boys $\geq 9$ years but $<10$ years old) on the basis of the appearance of pubertal signs (breast budding in girls and testis enlargement in boys), increased growth velocity, accelerated bone maturation, increased uterine and ovarian volume, and elevated $\mathrm{LH}$ response in a standard $\mathrm{GnRH}$ stimulation test $(\mathrm{LH}$ peak $>5 \mathrm{IU} / \mathrm{L}$; $\mathrm{LH}$ peak/FSH peak ratio $>0.6$ ) [13]. Testis enlargement is defined as testicular volume larger than $4 \mathrm{ml}$ measured by using Prader orchidometer. Blood samples for genetic analysis were obtained from the patients and their family members. All the participants were screened for variants in MKRN3. Among the MKRN3 negative patients, 19 samples (17 males and 2 females; 5 familial and 14 sporadic) were screened for variants in DLK1 gene.

2.3. Biochemistry. Blood samples were drawn from an antecubital vein between 8:00 and 10:00 AM and stored at $-20^{\circ} \mathrm{C}$ until analyses. $\mathrm{GnRH}$ stimulation tests were carried out as previously described [13]. Serum LH, FSH, estradiol, and testosterone levels were measured as previously described [13].

2.4. Variant Analysis. Genomic DNA was extracted from peripheral blood leukocytes from all patients following standard procedures. The exons of MKRN3 (NM_005664) and DLK1 (NM_003836.6) were amplified by PCR followed by automated sequencing of the products. If any variants were found in the probands, Sanger sequencing would also be performed in his or her parents. The primer sequences are 
listed in Supplemental Table 1. PCR was performed in an ABI 9700 Thermal Cycler under standard conditions. After purification using a QIAquick PCR Purification Kit (Qiagen), the products were directly sequenced onto an Applied Biosystems 3730xl automated sequencer (Life Technologies Corporation Carlsbad, CA, USA). Sequence comparisons and analyses were performed using the Phred-PhrapConsed program.

2.5. Sequence Retrieval, Homology Modeling, and In Silico Mutagenesis. The sequence information of MKRN3 was obtained from Uniprot database (http://www.uniprot.org, UniProtKB ID: Q13064). The division of domains and classification information were also from this database. We adopted the online server I-TASSER [14] for $a b$ initio modeling. We then received the initial overall structure of the wild-type MKRN3 for further analysis. Based on the obtained structure, we polished the domains whose local conformations were available, including three $\mathrm{C} 3 \mathrm{H} 1$ domains and one C3HC4 RING finger domain. Firstly, three temples (PDB ID: 1Z6U, 2Y43, and 1RGO) were retrieved via BLAST/PSI-BLAST. Furthermore, we polished the aforementioned four domains based on the initial structure using MODELLER V9.19 [15] platform. Finally, the further modification of the loop region in this structure was carried out, and we obtained the final 3D structure of MKRN3. We applied the VMD1.9.4 [16] platform for the 3D structure visualization and analysis.

\section{Results}

3.1. Sanger Sequencing and Familial Segregation Analysis. Sanger sequencing was used to identify variants of $M K R N 3$ genes of 173 ICPP patients and 43 early puberty patients. Four novel missense heterozygous variants (c.1138G $>$ A (p.Glu380Lys), c.1420T >A (p.Leu474Met), c.673C > G (p.Leu225Val), and c.1071C > G (p.Ile357Met)) and 2 synonymous heterozygous variants (c.336G > A (p.Gly112GLy) and c.870 T > C (p.Ile290Ile)) were detected in ICPP patients (Figure 2). All the 4 novel variants were found neither in ExAC nor in 1000G. Besides, these 4 variants were also not found in our local population database. A paternal mode of inheritance was observed in two familial cases with variants p.I357M (p.Ile357Met) and p.L225V (p.Leu225Val), respectively. A de novo missense variant E380K (p.Glu380Lys) was found in 2 sporadic and 1 familial cases of ICPP. The variant p.L474M (p.Leu474Met) was found inherited maternally; therefore, it is unlikely to be pathogenic. One synonymous heterozygous variant was detected in early puberty patients (c.396T $>$ A (p.Val132Val)). One polymorphism (c. 663C > T (p.Pro221Pro)) was found in 81 out of 216 patients, 8 of them were homozygous and others were heterozygous.

Variants of DLK1 gene were investigated in 19 ICPP or early puberty patients; 5 of them were familial and others were sporadic. Four heterozygous polymorphisms were found: (c.564T $>$ C (p.I188I) in 18 out of 19 patients, c.310G > A (p.V104M) in 2 out of 19 patients, c.699T > C
(p.C233C) in 2 out of 19 patients, and c. $404+221 \mathrm{C}>\mathrm{T}$ in 7 out of 19 patients).

3.2. Clinical and Biochemical Findings. Clinical and laboratory characteristics of patients studied are shown in Table 1. Clinical and hormonal data of patients with $M K R N 3$ variants are shown in Supplemental Table 2. All ICPP patients were treated by $\mathrm{GnRH}$ analogs (either triptorelin or Leuprorelin).

3.3. In Silico Structural Modeling and Analysis of the Variants. In the present study, we built the 3D structure of MKRN3 and analyzed the situations of the four clinically discovered nonsynonymous variants in the monolithic structure. Moreover, we also predicted the possible molecular mechanisms by which these variants lead to functional impact on the MKRN3 protein. As shown in Figure 3, we found that the protein was composed of five zinc-binding sites, including three $\mathrm{C} 3 \mathrm{H} 1$ zinc domains, one makorin-specific Cys-His zinc domain with MKRN family characteristic, and one C3HC4 RING finger domain. From this sequence model chart, we can easily find that I357 (Ile357) locates in the C3HC4 RING finger domain, while other variants L225 (Leu225), E380 (Glu380), and L474 (Leu474)) do not localize in any known zinc-binding region at the sequence level. To figure out the functional effect of these variants, we built 3D structure of protein MKRN3 using modeling software. From the structure diagram (Figure 4), we found that I357 and E380 are spatially extremely close to each other and both locate near the C3HC4 RING finger, MKRN-specific CysHis domain, and the third $\mathrm{C} 3 \mathrm{H} 1$ zinc-finger motif region. Meanwhile, both E380 and L474 are close to the third C3H1 zinc-finger domain spatially. Also, L225 residue is closed to the second $\mathrm{C} 3 \mathrm{H} 1$ domain and makorin-specific Cys-His domain.

\section{Discussion}

In the present study, we identified four novel MKRN3 missense variants in three familial and two sporadic cases. According to the ACMG guideline, the variants E380K and I357M were classified as likely pathogenic, and the variants L474M and L225V were classified as variants of uncertain significance (VUS). No pathogenic variants were found in DLK1 in our patients.

MKRN3 locates in the critical region of Prader-Willi syndrome (PWS) and is maternally imprinted. Normally, only the paternal allele is expressed, and the maternal allele is methylated. Therefore, theoretically, CPP can be caused while pathogenic MKRN3 variants come from the patient's father or when there is maternal uniparental disomy or chromosomal translocations. The MKRN3 defects are relatively common in European and Latin American CPP patients [10], especially in familial [17] and male [18] ICPP patients. However, MKRN3 variants caused ICPP are not so common in eastern Asian, according to the results of the present study and previous publications $[19,20]$.

MKRN3 protein belongs to the makorin protein family, which is characterized by combination of zinc-fingers. The 

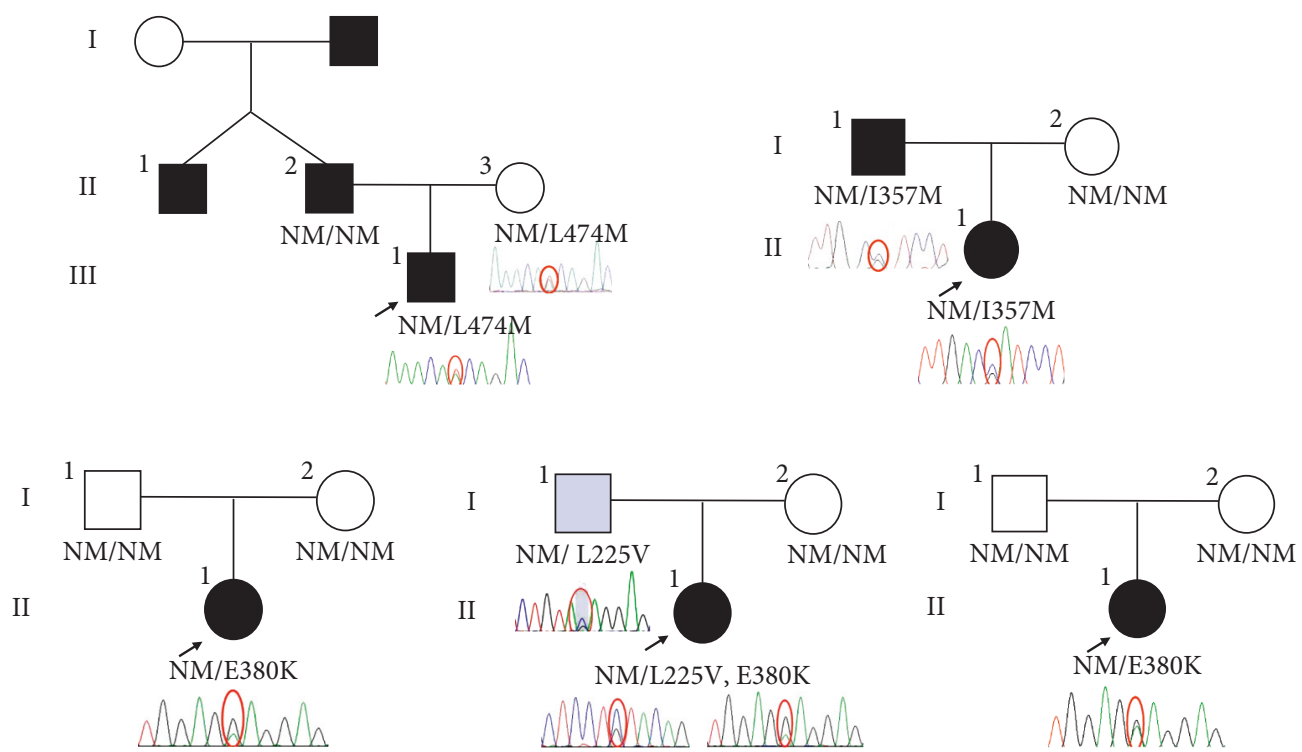

FIgURE 2: Pedigrees of patients with MKRN3 variants. Arrow, proband; square, male; circle, female; black symbols, subjects with idiopathic central precocious puberty; grey symbols, subjects with early puberty; WT, wild type. Roman numerals indicate the generation. Arabic numerals indicate the patients in each family.

TABLE 1: Clinical and biochemical features of the enrolled patients.

\begin{tabular}{lcccc}
\hline & \multicolumn{2}{c}{ CPP patients } & \multicolumn{2}{c}{ Early puberty patients } \\
& Boys & Girls & Boys & Girls \\
\hline Patients $(n)$ & 9 & 164 & 9 & 34 \\
Age at diagnosis (years) & $8.5(7.8-8.9)$ & $7.4(2.4-7.9)$ & $9.4(9.1-9.9)$ & $8.2(8.1-8.8)$ \\
BMI SD & $0.2(-0.5-2.0)$ & $0.4(-0.2-1.7)$ & $1.2(-0.2-2.8)$ & $0.4(-1.4-4.8)$ \\
Height SD & $1.2(-1.8-3.2)$ & $0.6(0.2-3.4)$ & $1.1(-2.1-2.8)$ & $0.7(-0.8-3.1)$ \\
Bone age (years) & $11.5(10.8-12.6)$ & $9.3(4.0-11.5)$ & $12.6(11.7-13.4)$ & $10.6(9.9-12.2)$ \\
Basal LH (UI/L) & $1.05(0.15-5.8)$ & $0.94(0.07-2.31)$ & $1.89(0.89-6.18)$ & $1.02(0.16-6.32)$ \\
Peak LH (UI/L) & $18.7(8.8-27.5)$ & $9.8(5.8-97.1)$ & $22.6(13.2-29.0)$ & $11.1(8.5-65.7)$ \\
Peak LH/FSH ratio & $1.8(0.7-2.5)$ & $1.1(0.6-2.68)$ & $2.6(1.1-3.8)$ & $1.3(0.7-3.2)$ \\
E2 for girls (pg/ml) & & $28.3(16.8-64.2)$ & & $48.9(19.2-59.6)$ \\
T for boys (ng/dl) & $85.4(45.6-185.8)$ & & & \\
\hline
\end{tabular}

Data are shown in median and range. BMI, body mass index; SD, standard deviation; LH, luteinizing hormone; FSH, follicle-stimulating hormone; E2, estradiol; T, testosterone.

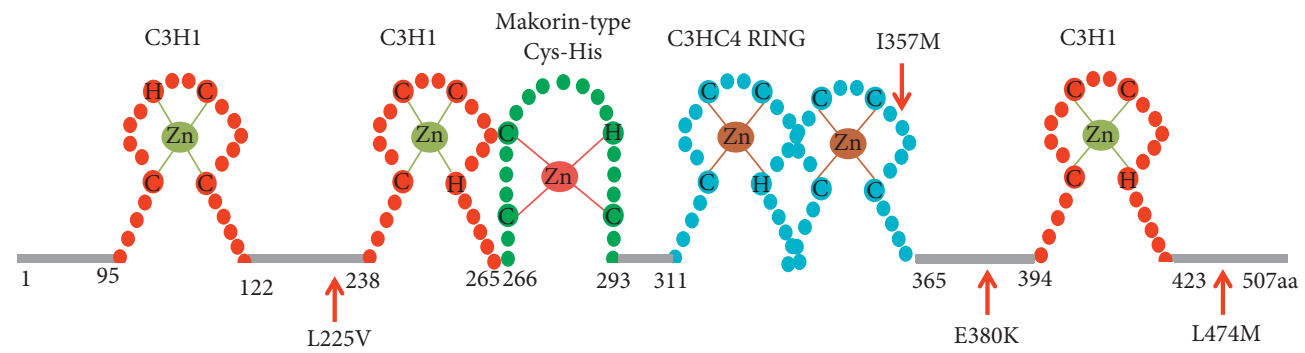

FIGURE 3: MKRN3 protein structure and variants identified in patients with central precocious puberty. Zn, zinc; $\mathrm{H}$, histidine; C, cysteine. The three $\mathrm{C} 3 \mathrm{H} 1$ zinc-finger motifs are shown in red, the C3HC4 RING finger motif is in blue, and the makorin-type Cys-His domain is shown in green. The numbers correspond to the amino acid positions in the protein. Red variant labels and arrows indicate the location of the missense variants.

Structure of MKRN3 contains two copies of a C3H1 motif in the N-terminal, followed by a makorin-type Cys-His domain, a C3HC4 RING zinc-finger, and a final C3H1 motif (Figure 3) [6]. Up to date, approximately 33 variants of
MKRN3 gene have been reported, among which 16 are missense mutation and 17 are null mutation. No hotspot region in MKRN3 gene has been discovered, but half of the premature termination variants locate between the two 


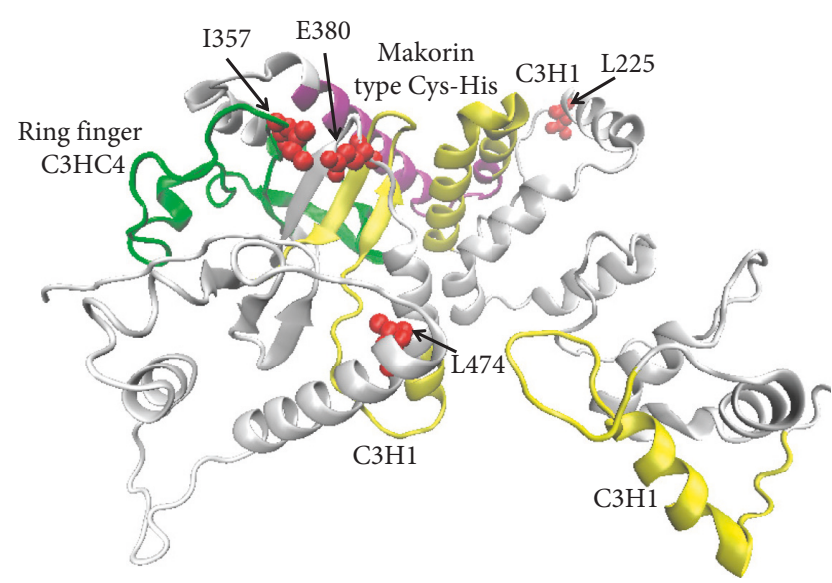

FIgURE 4: Native structure of the produced MKRN3 protein using ab initio modeling. The three $\mathrm{C} 3 \mathrm{H} 1$ zinc-finger motifs are shown in yellow, the C3HC4 RING finger motif is in green, and the MKRNspecific Cys-His domain is shown in purple. The four missense variant residues are highlighted with red spheres.

$\mathrm{N}$-terminal C3H1 zinc-finger motifs. Some missense variants were reported in more than one family. About half of the missense variants reported locate outside the functional domains. Since no functional studies are available, all these reported missense variants should be interpreted with caution. According to previous reports, frameshift or nonsense variants are likely to cause more severe phenotypes when compared to missense variants.

In the present study, we identified four novel variants, which were not found in ExAC, 1000G, or our local database. The variant E380K is de novo and predicted to be deleterious by multiple in silico tools. Besides, the patient's phenotypes and family history fit MKRN3 defect-caused CPP. The variant I357M is located in critical zinc-finger region and predicted to be pathogenic by several in silico tools. Moreover, the variant I357M is inherited from the proband's father with $\mathrm{CPP}$, which fits the specific inheritance pattern of MKRN3 mutation. The variant $\mathrm{L} 225 \mathrm{~V}$ is also inherited from the proband's father, who had early puberty instead of CPP. MKRN3 mutations may manifest less severe in males than in females, which was also reported in previous cases [21]. However, since L225V is predicted to be benign by multiple in silico tools, the pathogenicity of this variant is still in doubt. The variant L474M is also VUS since it is inherited from proband's mother instead of father.

Structural analysis reveals that the four variants discovered in the present study are located in different structural regions, which might have various functional effects on MKRN3 during biological processes. The change of Glu to Lys in E380K was suggested to have a notable effect on the function. Since the negatively charged amino acid glutamate is replaced by the positively charged amino acid lysine, the local physicochemical properties of the protein were completely changed, and the interactions with the surrounding amino acids may be altered (Figure 5). Considering the short distance between the sidechains, the native E380 may establish hydrogen bonds with the residues I357 and W377. However, after the mutation, the positively charged lysine

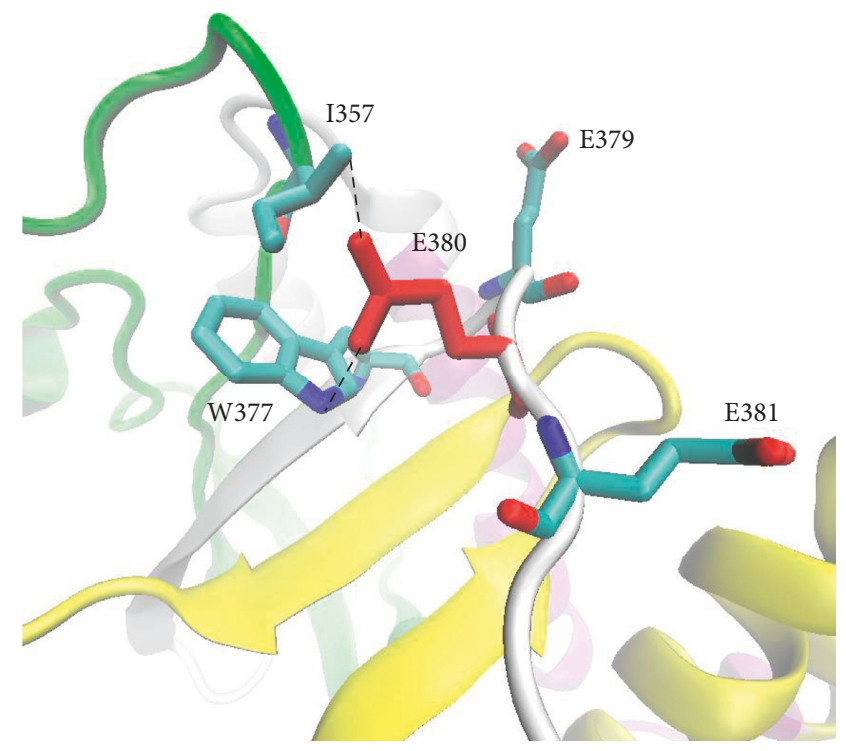

FIGURE 5: Interaction between E380 and surrounded residues.

will cause disruption of the two pairs of hydrogen bonds and will tend to form hydrogen bonds with E379 or E381 nearby, which in turn might affect the local structure stability, especially the stability of the zinc-binding region. It is highly possible that the local conformational distortions may directly influence the binding of ions. Structurally, the L225V, I357M, and L474M variants are all replacements between hydrophobic amino acids, which only alter the size of the side-chain groups. There are no significant differences in the interaction with surrounding amino acids. But considering the structures, as to the variants of I357M and L474M, the sulfur atom in the side-chain of the mutant methionine might interact with the $\mathrm{Zn}$ ion in the zinc-finger domain and change the original binding state between $\mathrm{Zn}$ ion and cysteine or interact with the cysteine in the protein, thereby influencing its original function.

Similar to $M K R N 3, D L K 1$ gene also locates in a maternal imprinting region located in $14 \mathrm{q} 32$, which is originally reported as a critical region of Temple syndrome. DLK1 encodes a transmembrane protein which is important for adipose tissue homeostasis and neurogenesis. The first case of DLK1 variant-caused CPP was just reported in 2017 [7]. According to the limited reports, $D L K 1$ variant may not be a common genetic cause of CPP. In the current study, we have not found any pathogenic variants of $D L K 1$ gene in our patients $[7,22]$.

Our study has several limitations. Firstly, the sample size was relatively small, and further studies with larger sample size are needed to confirm our findings. Secondly, we did not sequence KISS1 and KISS1R gene in our patients. Although KISS1 and KISS1R gene-caused CPP is extremely rare, there is still a possibility that the CPP in our patients are caused by defects of these 2 genes. Besides, the sample size of DLK1 gene sequencing is too small to reveal the real prevalence of these gene mutations in CPP patients. Lastly, since we only explored the pathogenicity of novel variants via ACMG guidelines and bioinformatic approaches, further functional 
studies are still needed to confirm the results of bioinformatic predictions.

In conclusion, we found 4 novel missense variants of $M K R N 3$ and no variant of DLK1 in Chinese ICPP patients. This study suggested that the prevalence of $M K R N 3$ variants might be low in Chinese ICPP patients. Multicenter studies with larger sample sizes are still needed to clarify the exact prevalence of MKRN3 and DLK1 gene defects-caused ICPP in Chinese population.

\section{Data Availability}

The clinical, genetic, and bioinformatic data used to support the findings of this study are included within the article.

\section{Conflicts of Interest}

The authors declare that they have no conflicts of interest.

\section{Authors' Contributions}

Ting Chen and Linqi Chen contributed equally to this study.

\section{Acknowledgments}

This study was supported by the National Natural Science Foundation of China (project code 81700793) and a Suzhou Science and Technology Development Project (project code SYS 201561) (awarded to Dr Ting Chen).

\section{Supplementary Materials}

Table 1: primer sequences of MKRN3 and DLK1 genes. Table 2: clinical characteristics of patients with MKRN3 mutations. TS, Tanner stage; BA, bone age; FSHb, basal FSH; LHb, basal LH; LHp, peak LH; UL, uterine length; TV, testicle volume. (Supplementary Materials)

\section{References}

[1] J.-C. Carel and J. Léger, "Precocious puberty," New England Journal of Medicine, vol. 358, no. 22, pp. 2366-2377, 2008.

[2] L. de Vries, A. Kauschansky, M. Shohat, and M. Phillip, "Familial central precocious puberty suggests autosomal dominant inheritance," The Journal of Clinical Endocrinology \& Metabolism, vol. 89, no. 4, pp. 1794-1800, 2004.

[3] C. B. G. Brook, P. Clayton, and R. Brown, Eds., Brook's Clinical Pediatric Endocrinology, John Wiley \& Sons, Hoboken, NJ, USA, 2009.

[4] M. G. Teles, S. D. C. Bianco, V. N. Brito et al., "AGPR54Activating mutation in a patient with central precocious puberty," New England Journal of Medicine, vol. 358, no. 7, pp. 709-715, 2008.

[5] L. G. Silveira, S. D. Noel, A. P. Silveira-Neto et al., "Mutations of the KISS1 gene in disorders of puberty," The Journal of Clinical Endocrinology \& Metabolism, vol. 95, no. 5, pp. 2276-2280, 2010.

[6] A. P. Abreu, A. Dauber, D. B. Macedo et al., "Central precocious puberty caused by mutations in the imprinted gene MKRN3," New England Journal of Medicine, vol. 368, no. 26, pp. 2467-2475, 2013.
[7] A. Dauber, M. Cunha-Silva, D. B. Macedo et al., "Paternally inherited DLK1 deletion associated with familial central precocious puberty," The Journal of Clinical Endocrinology \& Metabolism, vol. 102, no. 5, pp. 1557-1567, 2017.

[8] Y.-J. Rhie, K.-H. Lee, J. M. Ko, W. J. Lee, J. H. Kim, and H.-S. Kim, "KISS1 gene polymorphisms in Korean girls with central precocious puberty," Journal of Korean Medical Science, vol. 29, no. 8, pp. 1120-1125, 2014.

[9] L. Huijbregts, C. Roze, G. Bonafe et al., "DNA polymorphisms of the KISS1 3 ' untranslated region interfere with the folding of a G-rich sequence into G-quadruplex," Molecular and Cellular Endocrinology, vol. 351, no. 2, pp. 239-248, 2012.

[10] N. V. Ortiz-Cabrera, R. Riveiro-Álvarez, M. Á. LópezMartínez et al., "Clinical exome sequencing reveals MKRN3 pathogenic variants in familial and nonfamilial idiopathic central precocious puberty," Hormone Research in Paediatrics, vol. 87, no. 2, pp. 88-94, 2017.

[11] J. R. Perry, F. Day, C. E. Elks et al., "Parent-of-origin-specific allelic associations among 106 genomic loci for age at menarche," Nature, vol. 514, no. 7520, pp. 92-97, 2014.

[12] Z. Aycan, Ş. Savaş-Erdeve, S. Çetinkaya et al., "Investigation of MKRN3 variant in patients with familial central precocious puberty," Journal of Clinical Research in Pediatric Endocrinology, vol. 10, no. 3, pp. 223-229, 2018.

[13] T. Chen, H. Wu, R. Xie et al., "Serum anti-müllerian hormone and inhibin B as potential markers for progressive central precocious puberty in girls," Journal of Pediatric and Adolescent Gynecology, vol. 30, no. 3, pp. 362-366, 2017.

[14] J. Yang, R. Yan, A. Roy, D. Xu, J. Poisson, and Y. Zhang, "The I-TASSER suite: protein structure and function prediction," Nature Methods, vol. 12, no. 1, pp. 7-8, 2015.

[15] M. A. Martí-Renom, A. C. Stuart, A. Fiser, R. Sánchez, F. Melo, and A. Šali, "Comparative protein structure modeling of genes and genomes," Annual Review of Biophysics and Biomolecular Structure, vol. 29, no. 1, pp. 291-325, 2000.

[16] W. Humphrey, A. Dalke, and K. Schulten, "VMD: visual molecular dynamics," Journal of Molecular Graphics, vol. 14, no. 1, pp. 33-38, 1996.

[17] D. Simon, I. Ba, N. Mekhail et al., "Variants in the maternally imprinted gene MKRN3 are common in familial central precocious puberty," European Journal of Endocrinology, vol. 174, no. 1, pp. 1-8, 2016.

[18] D. S. Bessa, D. B. Macedo, V. N. Brito et al., "High frequency of $M K R N 3$ variants in male central precocious puberty previously classified as idiopathic," Neuroendocrinology, vol. 105, no. 1, pp. 17-25, 2017.

[19] W.-D. Lin, C.-H. Wang, and F.-J. Tsai, "Genetic screening of the makorin ring finger 3 gene in girls with idiopathic central precocious puberty," Clinical Chemistry and Laboratory Medicine, vol. 54, no. 3, pp. e93-e96, 2016.

[20] H. R. Jeong, H. S. Lee, and J. S. Hwang, "Makorin ring finger 3 gene analysis in Koreans with familial precocious puberty," Journal of Pediatric Endocrinology and Metabolism, vol. 30, no. 11, pp. 1197-1201, 2017.

[21] J. Känsäkoski, T. Raivio, A. Juul, and J. Tommiska, "A missense mutation in MKRN3 in a Danish girl with central precocious puberty and her brother with early puberty," Pediatric Research, vol. 78, no. 6, p. 709, 2015.

[22] A. Grandone, C. Capristo, G. Cirillo et al., "Molecular screening of MKRN3, DLK1, and KCNK9 genes in girls with idiopathic central precocious puberty," Hormone Research in Paediatrics, vol. 88, no. 3-4, pp. 194-200, 2017. 


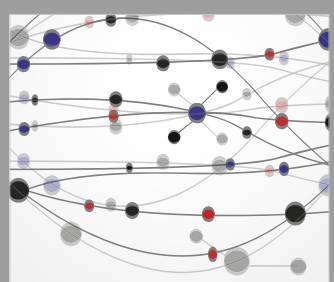

The Scientific World Journal
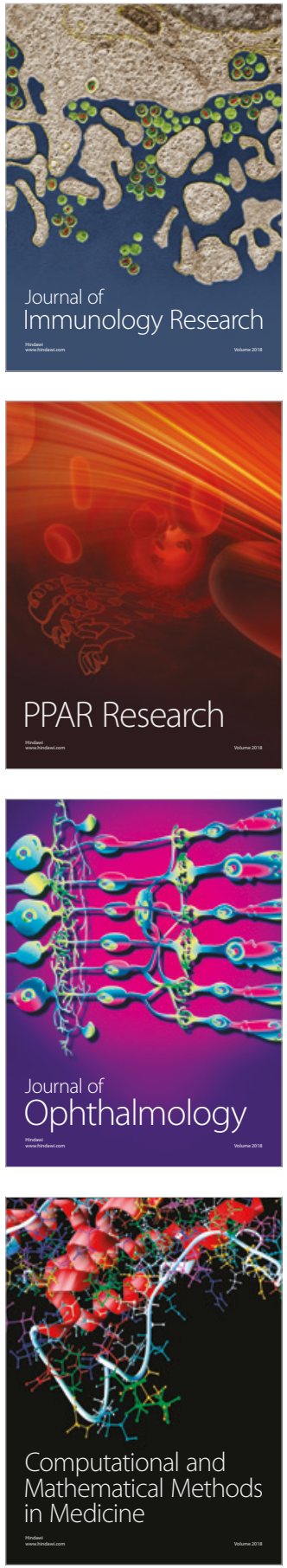

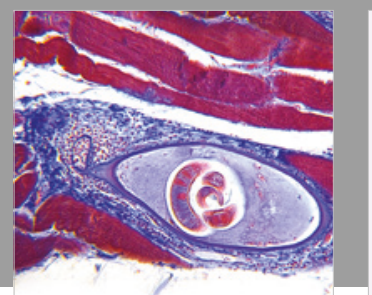

Gastroenterology Research and Practice

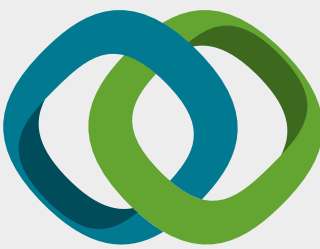

\section{Hindawi}

Submit your manuscripts at

www.hindawi.com
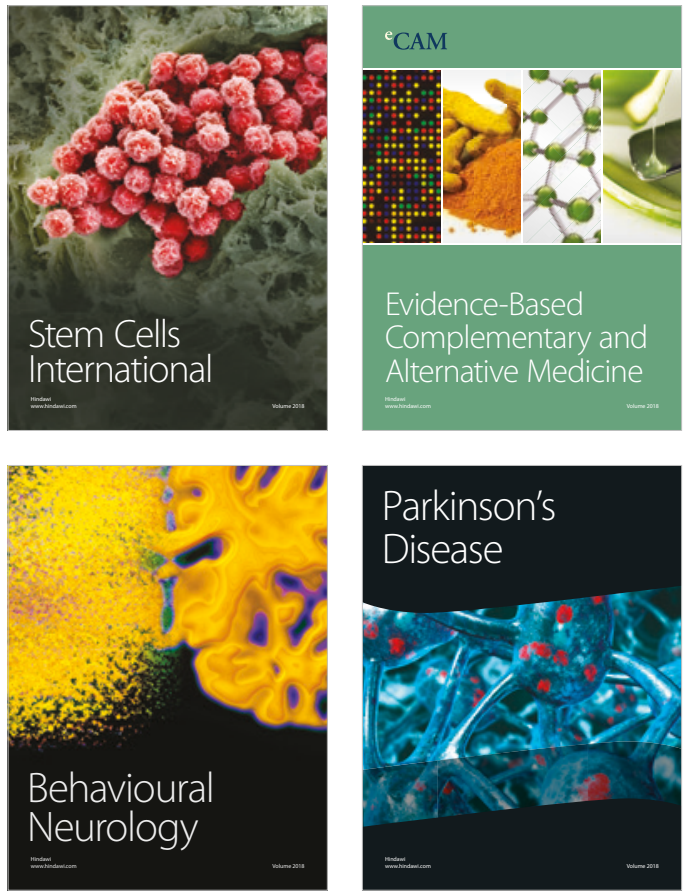

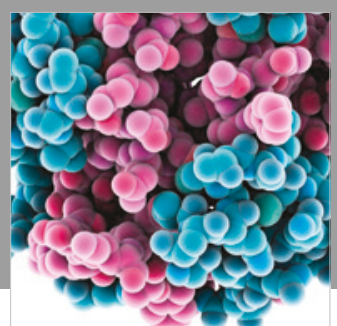

ournal of

Diabetes Research

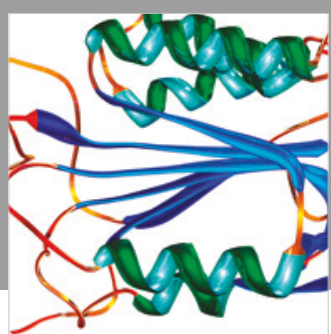

Disease Markers
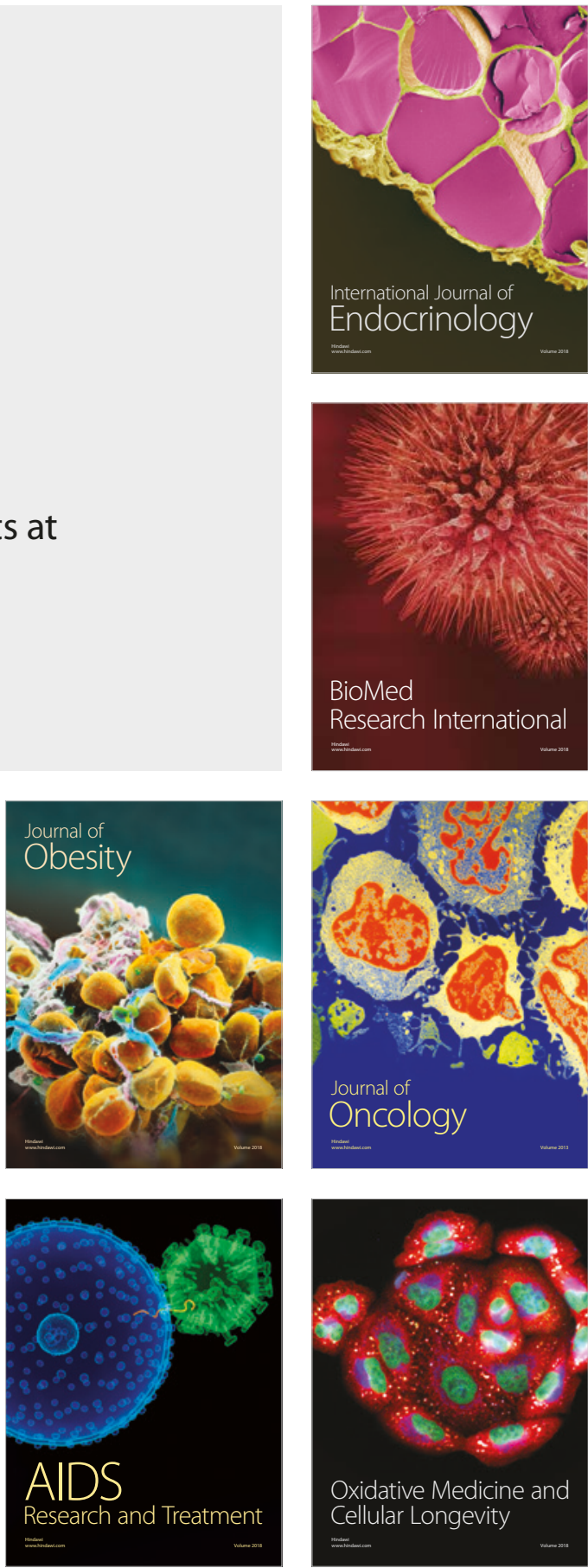